\title{
PECULIARITIES OF THE FATTY-ACID COMPOSITION OF BIRCH BUDS IN CONDITIONS OF YAKUTIA
}

\author{
(C) Perk A. A., Chepalov V. A., Petrov K. A.
}

Institute of Biological Problems of Cryolithozone, SB, Russian Academy of Science, Yakutsk, Russia

Birch buds in the form of decoction and tincture possess cholagogic, sudorific and diuretic properties, externally; they possess wound healing, antiseptic properties. The activity of the drugs may be connected with their fatty acid composition, depending on the time of buds collection. With the help of column chromatography, the quality composition and the content of fatty acids of the buds lipids $B$. platyphylla, which is one of the most frost resistant leaved species in the world, growing on the territory of the central Yakutia, was studied seasonally. All in all, 17 fatty acids are identified. In summer and in autumn, 11 and 9 fatty acids were depicted respectively, and in winter 16 fatty acids were discovered, and it is much more. The absolute content of fatty acids in buds constituted (summer-winter): $14.9+1.5-18.0+1.9 \mathrm{mg} / \mathrm{g}$ of the dry substance, the relative content of separate fatty acids: saturated-palmitic (C16:0) 23.7-24.4\%, stearin (C18:0) 4.1-2.7\%, arachidic (C20:0) 5.1-2.8\%, begenic (C22:0) $10.3-4.8 \%$ and unsaturated-oleinic (C18:1) 4.5-4.4\%, linolenic (C18:3)20.0-10.9\%. After fall of the leaves, recession was marked twice the level of saturated long-chained fatty acids $(C>22)$ and increase of the part of fatty acids on $18 \%$. Among unsaturated fatty acids in birch, the clear dynamics had two acids- linoleic, the relative content of which grew on $14 \%$ in autumn and in winter on $58 \%$, and the linolenic one, the content of which, on the contrary, decreased almost twice. In winter, minor fatty acids with odd quantity of atoms were found- pentadecane (C 15:0), margaric (C17:0) and unsaturated fatty acids of the eicosane row (C20:1-3). Thus, obtained data on seasonal dynamics of quality and quantity composition of buds lipids B. plathylla fatty acids, justify to considerable increase in winter of the common level of polyunsaturated fatty acids, mainly on the account of the linoleic acid. Probably, plants, enduring extreme low temperature stress in the conditions of Yakutia (up to $-60^{\circ} \mathrm{C}$ ), capable to accumulation of the large quantity of the polyunsaturated fatty acids row, possessing F-vitamin activity, in comparison with similar species of more moderate places of habitation, and it is necessary to take it into account while making drugs from them.

\section{MOLECULAR CLONING AND EXPRESSION OF O-METHYLTRANSFERASES FROM OPIUM POPPY}

\section{(C) Pongkitwitoon Benyakan, Sakamoto Seiichi, Sasaki Kaori, Tanaka Hiroyuki, Morimoto Satoshi}

\section{Graduate School of Pharmaceutical Sciences, Kyushu University, Fukuoka, Japan}

Papaver somniferum (opium poppy) has been known to be the essential source for several pharmaceutically important benzylisoquinoline alkaloids (BIAs) such as morphine, codeine, sanguinarine and papaverine. $O$-Methylation is regarded as an important reaction step in BIAs biosynthesis. Several cDNAs encoding proteins that catalyze these reactions have been reported, whereas many steps are still unclear. In this study, we attempt to clone P.somniferum $O$-methyltransferases to better understand biosynthetic pathways of BIAs in this plant. CDNA fragments encoding $O$-methyltransferase were amplified by homologybased RT-PCR using degenerate primers designed from conserved sequences of reported P.somniferum
O-methyltransferases. 3'- and 5'- end regions of cDNAs were obtained by rapid amplifications of cDNA ends. The full length cDNAs were then engineered to be expressed in silkworm using a rapid Bombyx mori nucleopolyhedrovirus (BmNPV) bacmid DNA system. Four cDNAs encoding polypeptides named PSOMT1, PSOMT2, PSOMT3, and PSOMT4, which consist 355, 356, 354, and 358 amino acids respectively, were cloned. Conserved motifs including SAM binding region, catalytic histidine, and metal binding region were found. The cloned cDNAs encoding these peptides are successfully expressed in the hemolymph of silkworm larvae. The purified PSOMTs are to be characterized as further study. 